\title{
Inferior Vena Cava Occlusion
}

National Cancer Institute

\section{Source}

National Cancer Institute. Inferior Vena Cava Occlusion. NCI Thesaurus. Code C35298.

Blockage of the lumen of the inferior vena cava. 